\title{
A Study on Identification and Characterization of Dengue Infection in a Tertiary Care Hospital
}

\section{C.K. Bhuvaneswari* and Eunice Swarna Jacob}

Department of Microbiology, Thanjavur Medical College, Thanjavur-613004, Tamilnadu, India

*Corresponding author

A B S T R A C T

\begin{tabular}{|c|c|}
\hline & \multirow{4}{*}{$\begin{array}{l}\text { In order to provide timely information for the effective treatment of dengue } \\
\text { patients, it is important to establish diagnosis of Dengue virus infection } \\
\text { during the first few days of clinical symptoms. Furthermore, determination } \\
\text { of the serotype of Dengue virus is also important for the surveillance of } \\
\text { Dengue Fever. If not diagnosed earlier the infection will lead to increased } \\
\text { mortality and morbidity. Hence, the aim is to diagnose Dengue infection, its } \\
\text { local epidemiology, seroprevalance and determination in Thanjavur district } \\
\text { by viral isolation using cell line, serological tests, and molecular methods } \\
\text { and genome sequencing. }\end{array}$} \\
\hline & \\
\hline & \\
\hline & \\
\hline
\end{tabular}

\section{Introduction}

Dengue infection is a systemic and dynamic disease. Infection with Dengue virus causes spectrum of clinical illness ranging from unapparent infection to mild nonspecific viral syndrome to classical Dengue fever to severe and fatal haemorrhagic disease. It occurs in tropical areas and affects up to 100 million people each year, including 500,000 cases of Dengue hemorrhagic fever and about 30,000 deaths, mostly among children. Dengue fever is a viral disease transmitted by mosquitoes (arthropod-borne) and caused by four serotypes of Dengue virus (DEN-1, DEN-2, DEN-3, and DEN-4). After an incubation period of about 4 to 10 days the illness begins abruptly and is followed by three phases namely febrile, critical and recovery.
Dengue Haemorrhagic Fever (DHF) is a severe form of Dengue viral infection characterized by sudden onset of fever, usually of 2 to 7 days duration with nonspecific signs and symptoms. The critical stage of DHF occurs either 24 hours before or 24 hours after the temperature falls to normal or below normal. During this time haemorrhagic manifestations usually occur, and signs of circulatory failure may appear (WHO 1997). Classical DHF with a capillary leak syndrome that has a unique immuno pathological basis associated with heterologous antibody-dependent enhancement of viral infection of cells of the mononuclear phagocyte lineage. Infection of these cells stimulates the release of vasoactive 
mediators that apparently causes increased vascular permeability if not promptly detected and corrected, can lead to hypovolemia, shock and death (Blacksell et al., 2007; McPride and Bielefeldt-ohmann 2000).

Dengue virus is an enveloped positive-sense RNA virus. The genomic RNA is approximately $10.7 \mathrm{~kb}$ in length and is composed of three structural protein genes that encode for necleocapsid or core protein (C), a membrane-associated protein (M), an envelope protein $(\mathrm{E})$ and seven non-structural protein genes including NS1 protein. NS1 is a highly conserved glycoprotein which appears essential for viral replication (David 2008). Dengue virus attachment to the host cell surface is mediated by the viral attachment protein (VAP), which seems to be the glycoprotein E present on the viral membrane (Robert Anderson et al., 1992). Dengue virus can infect its host cells through the binding of virus complexes to the Fc receptor or through the direct interaction of viral proteins with a specific host cell receptor (Ichiro Kurane et al., 1991). The acquired immune response following Dengue infection consists of the production of $\operatorname{IgM}$ and $\operatorname{IgG}$ antibodies primarily directed against the viral envelope proteins. The immune response varies depending on whether the individual has a primary (first Dengue or other Flavivirus infection) or a secondary (had Dengue or other Flavivirus infection in past) Dengue infection.

Dengue infection induces a lifelong protective immunity to the homologous serotype only and there is no cross-reactive immunity to the heterologous serotype. Instead it has been generally accepted that secondary or multiple Dengue virus infection is a major risk factor for Dengue Haemorrhagic Fever / Dengue Shock Syndrome, in addition to other factors, such as viral virulence and host genetic background.14 Therefore, differentiation of primary versus secondary or multiple Dengue viral infection is essential in analyzing data for epidemiological, pathological, clinical and immunological studies (Anoop et al., 2010).

Many factors including unprecedented population growth, increased population density, unplanned and uncontrolled urbanization, increased global travel, increased density of the vector mosquito, infestation of new geographical areas by vector mosquitoes, warm and humid climate and water storage pattern in houses that promote the spread of Dengue virus have all contributed to the remarkably increased incidence of Dengue infection in India during the past few decades (Anita Chakr Howe 1977;WHO 1997 and Anoop et al., 2010). Timely and correct diagnosis is very critical for patient management.

Dengue can be diagnosed by isolation of the virus, serological tests, or molecular methods. Diagnosis of acute (on-going) or recent Dengue infection can be established by testing serum samples during the first 5 days of symptoms and/or early convalescent phase (more than 5 days of symptoms)(Vaughn et al., 1997). Acute infections can also be laboratory confirmed by identification of Dengue viral antigen or RNA from the serum of the infected individual / autopsy tissue specimens by immunofluorescence or immune histochemical analysis, or by sero conversion from negative to positive IgM antibody to Dengue or demonstration of a fourfold or greater increase in IgG antibody titers in paired (acute and convalescent) serum specimens (Gubler et al., 1999).

For virus detection, viral isolation by cell culture remains the "gold standard". Viral isolation methods are time consuming and usually take a week. Different types of polymerase chain reactions (PCR) like reverse-transcription PCR (RT-PCR) real- 
time PCR and nested or hemi-nested PCR are used for detecting genomic sequence for serotyping (Seah et al., 1996 and Sudiro et al., 1997). Use of PCR techniques is a quick and sensitive method for detecting Dengue virus and has replaced viral isolation techniques (Gurukamal et al., 2009). Use of serological methods by detecting viral antiIgM and/or anti-IgG can give false positive results due to extensive antigenic crossreactivity among Flavivirus as well as between different Dengue virus serotypes. Recently IgM and IgG capture ELISA have been modified into immunochromographic formats in which the result of the assay is rapid and can be visualized by the appearance of a colour band (visible to the naked eye) making the interpretation easier (Cardosa and Tio 1991). However because of their format these kits are most suitable for individuals or small numbers of patient (Aikat et al., 2011; Vaughn et al., 1998).

In order to provide timely information for the care of the patient, it is important to establish a diagnosis of Dengue virus infection during the first few days of clinical symptoms. Furthermore, determination of the serotype of Dengue virus is also important for the surveillance of Dengue Fever (WHO 2009).

All four Dengue virus serotypes have been reported periodically in this country, but Dengue virus-2 has usually been the main etiological agent in DF and DHF outbreaks and has emerged as the predominant serotype (Parida et al., 2002).

The introduction of a serotype never isolated in a region previously will result in rapid spread and land in to an epidemic of Dengue Fever. In such cases surveillance measures should be increased (WHO 2005). Southern India also has a history of outbreaks of Dengue viral infection however, the responsible serotypes are not well known.

\section{Materials and Methods}

This study was conducted among the PUO cases attending the Medical OP at Thanjavur Medical College Hospital and Paediatric Medicine OP at Government Raja Mirasudhar Hospital, Thanjavur. The study population includes 230 suspected cases of Dengue Fever with 3-10 days duration.

The suspected samples were screened by Rapid DUO cassette method (Immunochromatography) for simultaneous detection of Dengue virus NS1 Ag and Differential $\operatorname{IgM} / \operatorname{IgG}$ antibodies by $\mathrm{SD}$ Dengue NS1 Ag $+\mathrm{Ab}$ Combo Kit at Department of Microbiology, Thanjavur Medical College Thanjavur. IgM capture ELISA was done for all the suspected samples, at Department of Microbiology, Thanjavur Medical College Thanjavur. RTPCR was done for 20 Dengue positive samples at King Institute of Preventive Medicine, Guindy Chennai. Isolation of Dengue virus by inoculating the 9 serum samples including $5 \mathrm{PCR}$ positive sera in C6/36 cell line and observed the cytopathogenic effects, by using inverted microscope. Confirmation of the Dengue Antigen titre is by Heamagglutination Assay. Genomic Sequencing was done for the 5 PCR positive samples

\section{Sample collection}

Blood samples were collected, after getting oral consent from the suspected cases of Dengue. $5 \mathrm{ml}$ of whole blood was drawn from the intravenous route with aseptic precautions and transferred to sterile screw caped vials. The caps were fixed with adhesive tape to prevent leakage during transport. The blood samples were transported to the lab immediately in an ice box with proper labeling (name of the patient, identification number and date of collection) 


\section{Processing of samples}

The samples were taken to the laboratory immediately and they were allowed to clot by placing it in a rack at room temperature (20$25^{\circ} \mathrm{C}$ ) for at least 30 minutes. After that the serum was separated by centrifugation. The clarified serum was then transferred to a sterile vial, and was stored at the required temperature depending upon the usage [Short term storage at $+4^{\circ} \mathrm{C}$ and long term storage at $\left.-70^{\circ} \mathrm{C} 129\right]$.

\section{The dengue NS1 Ag rapid test}

The Dengue NS1 Ag rapid test cassette is an in-vitro Immunochromatographic one step assay designed for the qualitative determination of Dengue virus NS1 antigen in human serum, plasma or whole blood for the diagnosis of early acute Dengue infections. The Dengue NS1 Ag test device contains a membrane strip with a result window which has 2 pre-coated lines, one with anti-Dengue NS1 Ag capture on test band region., "T" (NS1Ag Test Line) and the other "C" (Control line). Both the Test Line and the Control Line in result window are not visible before applying any samples.

\section{Dengue IgM capture ELISA}

IgM antibodies if present in the patient's blood will be captured by Antihuman IgM. ( $\mu$ Chain specific) that are coated on to the solid surface (wells).

When DEN antigen is added it will bind to capture $\operatorname{IgM}$, if the $\operatorname{IgM}$ and antigen are homologous. Unbound antigen is removed during the washing step. In the subsequent step Biotinylated Flavivirus cross reactive monoclonal antibody (Hx-B) is added followed by Avidin-HRP. Subsequently, by adding substrate / chromogen, visible colour change will be observed. The reaction was stopped by $1 \mathrm{~N} \mathrm{H} 2 \mathrm{SO} 4$. The intensity of color/optical density is monitored at $450 \mathrm{~nm}$ by using ELISA Reader. OD readings are directly proportional to the amount of Dengue virus specific IgM antibodies in the sample.

\section{Isolation of Dengue virus}

For Isolation: C6 / 36 (mosquito cell line of Aedes albopictus) were obtained from NCCS and used for the study. After initiation of a cell culture, medium was changed periodically and followed eventually by subculture when the cells are proliferating. Sub-culture was initiated when the cell density reaches a level such that all the available substrates were occupied or when the cell concentration exceeds the capacity of the medium.

Identification of viral genome by conventional Reverse Transcriptase PCR (RT-PCR)

Dengue RNA was extracted and PCR was performed following were the primer sequences used for carrying out the polymerase chain reaction:

Dengue 1

MD1 - 5'

TCAATATGCTGAAACGCGAGAGAAAC CG 3'

MTS1-2 - 5'

CCCGTAACACTTTGATCGCT 3'

Dengue 2

MD $1-5^{\prime}$

TCAATATGCTGAAACGCGAGAGAAAC

CG 3'

TS-2 - 5' CGCCACAAGGGCCATGAACAG 3' 3. 
Dengue 3

MD 1 - 5'

TCAATATGCTGAAACGCGAGAGAAAC CG 3'

TS-3 - 5'

TAACATCATCATGAGACAGAGC 3'

Dengue 4

MD 1 - 5'

TCAATATGCTGAAACGCGAGAGAAAC CG 3'

\section{MTS4-5 - 5' TTCTCCCGTTCAGGATGTTC $3^{\prime}$}

The PCR product was run on the agarose gel electrophoresis and the bands were determined. After visualization in the agarose gel, the PCR products were purified using gel extraction column and subjected to DNA sequencing from synergy scientific Bangalore.

Identified Dengue viral strain was analysed together with various global reference sequences of different genotypes available from Genbank. The nucleotide sequences of complete $\mathrm{E}$ genes of DENV strain was aligned edited and analyzed using Cluster W Software and Phylogenetic tree was constructed. In order to find out the nucleotide sequence homology of the etiological agent, the nucleotide sequences thus obtained from the commercial sequencing service was further subjected for ClustalW analysis in NCBI databank.

\section{Results and Discussion}

The incidence of Dengue infection in this study, over a period of one year was $0.6 \%$ (Table 1) and the burden of disease was 1 out of 167 patients. The screening of 230 suspected samples for the presence of NS1 antigen and $\operatorname{IgM} / \mathrm{IgG}$ antibodies by SD Bioline Dengue DUO combikit showed 80 cases $(34 \%)$ were positive for both NS1 antigen and antibodies (Table 4). In the present study it was found that 49 out of 80 $(61.25 \%)$ of the affected population were males and 31 out of $80(38.75 \%)$ were females (Table 2). The analysis of the suspected samples for the presence of NS1 antigen and $\operatorname{IgM} / \mathrm{IgG}$ antibodies by $\mathrm{SD}$ Bioline Dengue DUO combikit showed that out of 80 samples $9(11.25 \%)$ were NS1 antigen positive, $58(72.5 \%)$ were $\operatorname{IgM}$ antibodies positive, $20(25 \%)$ were $\mathrm{IgG}$ antibodies positive and $30(37.5 \%)$ were both IgM and IgG positive (Table 5). All the 230 samples were further analysed according to the clinical manifestation and it was found that among the Dengue positive cases 58 cases $(72.5 \%)$ were Dengue fever, 22 cases (27.5) were Dengue haemorrhagic fever and there was no case with Dengue shock syndrome.

Twenty serum samples (9 NS1 positive and 11 IgM Positive - samples collected within 6 days from the onset of illness and platelet count being less than 1 lakh) collected from suspected patients were tested by RT-PCR for the confirmation of Dengue infection. Viral RNA was isolated from each of these samples and was subjected to the RT-PCR assay. Of these 20 samples, only five samples answered positive. All the five PCR positive cases were NS1 antigen Positive Samples.

All the 5 PCR positive samples were subjected to Molecular Typing of Dengue virus namely D1, D2, D3, and D4. All the five samples were positive for both Dengue virus sero type 1 and sero type 4 by the RT-PCR method. This was confirmed by obtaining the correctly sized DNA product (D1-208 bp, D2119 bp, D3- 288 bp, D4-260 bp) for each of these samples after amplification with 
consensus primers of Dl and D4 (Figure 1). This signified that the presently circulating strain of Dengue virus in Thanjavur is DENGUE 1 and DENGUE 4 (Table 6).

The high prevalence of Dengue cases at Thanjavur in the recent years makes it necessary to evaluate the sero positivity, serotyping and strains of Dengue in our hospital. The Dengue infection was most prevalent during post-monsoon season and is more of male preponderance. The Dengue fever and Dengue Haemorrahagic Fever is present in Thanjavur and no Dengue Shock Syndrome cases. Fever was the most common presenting symptom (100\%), followed by myalgia $(87 \%)$ and headache $(68 \%)$ in Dengue infection. The common haemarrhagic manifestations among Dengue patient were gum bleeding and melena. The Dengue virus 1 and 4 strains are prevalent in Thanjavur. Though the number of DENV -1 and 4 Isolates recovered from the present study was less, the circulation of Dengue virus isolates particularly DENV-1 and 4 in Southern India are noteworthy.

The suspected samples for the presence of NS1 antigen signify that early detection is possible with NS1 antigen and $\mathrm{IgM} / \mathrm{IgG}$ antibodies detection for diagnosis of infection.

The Dengue IgM seropositivity among the suspected cases indicates active Dengue virus activity. Increase in the probable secondary infection especially in a country like ours where multiple serotypes are prevalent raises concern over probable increase in the incidence of the more serious DHF/DSS. The need of the hour is to characterise the circulating serotype in our area and to understand the evolutionary process influencing the Dengue virus, as this is expected to impact on vaccination strategies for future
The circulation of all 4 serotypes of Dengue viruses has already been documented in Tamilnadu, and the present findings indicate the possible spread of the potent Dengue virus serotype 1 and 4 to newer areas. Further studies are needed to continuously monitor the movement and behavior of the virus strain in southern India.

\section{Acknowledgments}

I am very thankful to our respected Dean Dr. T.B. Umadevi, M.D Thanjavur Medical College, Thanjavur for permitting me to carry out this study. I wish to express my sincere thanks to Dr. P. Gunasekaran., M.D., M.B.A Director i/c and Dr. Kaveri krishnasamy M.D., Dy. Director, Department of Virology, King Institute of Preventive Medicine and Research for permitting me to do the research work in KIPM, Chennai.

\section{References}

Aikat, A., Sarkar, A., Tudu, N., Moitra, S., Pal, P.P. Sarkar, T.K., 2011. Diagnostic dilemma of dengue fever: how much reliability is there in rapidity $\mathrm{J}$. Ind. Med. Assoc, 109 : 543-545

Anita chakr Howe, G. M., 1977. A world geography of human diseases. Academic Press, Inc., New York, N.Y.

Anonymous, 1997. Dengue haemorrhagic fever: diagnosis, treatment, prevention and control. World Health Organization, Geneva, Switzerland.

Anoop, M., Issac, A., Mathew, T., Philip, S., Abdul Kareem, N., Unnikrishnan, R. and Sreekumar, E., 2010.. Genetic Characterization of dengue virus serotypes causing concurrent infection in an outbreak in Ernakulam, Kerala, South India. Ind. J. Experi. Biol, 48: 81-857

Blacksell, S.D., Bell, D., Kelley, J., Mammen, M.P., Gibbons, J.R.V., Jarman, R.G., Vaughn, D.V., Jenjaroen, K., Nisalak, A., Thongpaseuth, S., Vongsouvath, M., Davong, V., Phouminh, P., Phetsouvanh, R., Day, N.P.J. and Newton, P.N., 2007. 
Prospective study to determine accuracy of rapid serological assays for diagnosis of acute dengue virus infection in laos. Clin. Vacci. Immun, 1458-1464.

Cardosa, M.J., and Tio, P.H, 1991. Dot enzyme immunoassay: an alternative diagnostic aid for dengue fever and dengue haemorrhagic fever. Bull.Worl. Healt. Organ, 69: 741745.

David, S., Gppdsell July 2008. Molecule of the month: dengue virus.

Goro Kuno, serodiagnosis of flaviviral infections and vaccinations in humans.

Gubler. D. J., Meltzer. M, Impact of dengue/dengue hemorrhagic fever on the developing world, Adv Virus Res. (1999), 53, 35-70.

Gurukumar, K.R., Priyadarshini, D., Patil, J.A., Bhagat, A., Singh, A., Shah, P.S. and Cacilia, D., 2009. Development of real time PCR for detection and quantization of dengue viruses. MioMed Cent. Virol, J, 6:10.

Ichiro Kurane, Bruce L. Innis, Suchitra Nimmannitya, Ananda Nisalak, Anthony Meager, Jurand Janus \& francis A. Ennis. Activation of Lymphocytes in Dengue virus Infection. The American Society for Clinical Investigation, Inc. Volume 88, 1991, 1473-1480.

McBride, W. J. H., and H. Bielefeldt-Ohmann, 2000. Dengue viral infections; pathogenesis and epidemiology. Microbes Infect. 2:1041-1050.

Parida, M.M., Dash, P.K., Upadhyay, C., Saxena, P. and Jana, A.M., 2002. Serological and virological investigation of an outbreak of dengue fever in Gwalior, India. Ind. J Med Res, 116: 248-254.
Robert Anderson, Alan D. King, \& Bruce L. Innis. Correlation of $\mathrm{E}$ protein binding with cell susceptibility to dengue 4 virus infection. Journal of General Virology (1992), 73, 2155-2159.

Seah, C. L. L., Chow, V. T. K. Tan, H. C. and. Chan, Y. C 1995. Rapid single-step RTPCR typing of dengue viruses using NS3 gene primers. J. Virol. Methods 51:193200.

Sudiro, T.M., Ishiko, H. Green, S. Vaughn, D. W. Nisalak, A. Kalayanarooj, S. Rothman, A. L Raengsakulrach, B. Janus, J. Kurane, I. and Ennis. F. A. 1997. Rapid diagnosis of dengue viremia by reverse transcriptase polymerase chain reaction using 3_noncoding region universal primers. Am. J. Trop. Med. Hyg. 56:424-429.

Vaughn, D.V., Nisalak, A., Kalayanarooj, S., Solomon, T., Minh Dung, N., Cuzzubbo, A. and Devine, P.L., 1998. Evaluation of a rapid immunochromatographic test for diagnosis of dengue virus infection. J. Clin. Microbiol, 234-238

Vaughn, D.W, Green, S., Kalayanarooj, S., Innis, B.L, Nimmannitya, S, Suntayakom S, Rothman AL, Ennis FA, Nisalak A, 1997. Dengue in the early febrile phase: viremia and antibody responses. J Infect Dis 176: $322-330$.

World Health Organization. 2005. Epidemic and pandemic alert and response: impact of dengue. World Health Organization, Geneva, Switzerland. [Online.] http://www.who.int/csr/disease/dengue/imp act/en/index.html.

World Health Organization.2009. Guidelines for diagnosis, treatment prevention and control new edition.

\section{How to cite this article:}

Bhuvaneswari, C.K. and Eunice Swarna Jacob. 2017. A Study on Identification and Characterization of Dengue Infection in a Tertiary Care Hospital. Int.J.Curr.Microbiol.App.Sci. 6(10): 1827-1833. doi: https://doi.org/10.20546/ijcmas.2017.610.220 